\title{
THE RELATIONSHIP BETWEEN SELF-ESTEEM AND AGGRESSIVE BEHAVIOR AMONG PRETEENS
}

\author{
Irina BANU, \\ Prof. School Counseling Teacher CMBRAE Bucharest, \\ PhD. Student, University of Bucharest, \\ Romania \\ E-mail: tmirina@yahoo.com
}

\begin{abstract}
Students' aggressive behavior generates significant difficulties in the educational environment. It is essential to pay close attention to these issues as their effects often extend beyond short-term disturbances. The school tried to react at the students'aggressiveness problems by implementing intervention programs, counseling programs. These interventions can reduce aggressive behaviors by boosting the social skills, by improving emotional adjustment and by solving problems encountered in a social context. Such intervention programs already exist in schools which is important to mention. Despite that, more and more students are in need of personal development and unfortunately there is only one available specialist in charge of these programs. Developing a high self-esteem can help the student to overcome his/hers aggressiveness through experimenting emotions like acceptance and understanding. As a consequence, it is mandatory to use counseling programs in order to reduce and overcome these behaviors. Throughout my life experience, I came to the conclusion that there is a direct link between low self-esteem and aggressive behavior which needs to be investigated. Within a focus group, there were observed several discontents from the students' side such as: not being listened to, not being encouraged, not being appreciated or not being valued. All the students questioned said that if they were being listened to, encouraged, appreciated or valued, they would be more balanced. The current research analyzed the relationship between self-esteem and aggressive behavior of preadolescent students, the main objective being to highlight the need to develop and implement some methods that are required for growing the self-esteem.
\end{abstract}

Keywords: aggression; self-esteem; socio-emotional development;

\section{INTRODUCTION}

Long-term aggression can lead to disturbing relationships with others, the latter refusing to communicate and interact with the aggressive ones. Excluding the aggressive students from groups could lead to a decrease in their self-esteem. Therefore, developing high self-esteem could support the aggressive student in diminishing these aggressive behaviours by experiencing feelings of acceptance and understanding. Consequently, it is necessary to intervene in reducing these behaviours through counselling programs.

The central premise of the intervention program asserts that thoughts influence how children feel and act. The behaviour in question is defined not only by how we understand, process and interpret the information but also by how we express our emotions and how we react on their basis. 


\section{PROBLEM STATEMENT}

How is self-esteem formed and what makes the child lose it? What exactly affects children's' self-esteem and what exactly helps in increasing it?

The child's self-esteem is built, has an attachment and is strongly influenced by mother's self-esteem. It is important to treat a child with love and acceptance, autonomy and emotional support.

School is, also, a very important context that parents can no longer fully control and where a child's self-esteem can be strongly affected. The child lacks self-esteem in school with relation to several aspects, either to the fact that they cannot cope with the volume of work or to relationships with colleagues or with teachers. In the school setting, the aggressive behavior was positively correlated with a low level of academic involvement, the number of friends in the classroom, the perception of the level of support of the teacher and the positive attitude towards the school (Estevez et al., 2018).

Among the social factors that contribute to the aggressive behavior of children and students, the circle of friends is one with high impact. There are two main ways in which the experiences with the circle of friends are related to the development of aggressive or, worse, violent behaviors. On the one hand, colleagues or friends "may cause psychological or physical violence in the form of rejection or victimization, which may accentuate aggressive behavior in some children, thus contributing to the cycle of violence" (Vitaro et al., 2007, 361). On the other hand, the group of colleagues and friends can encourage aggressive behaviors, encouraging them and offering a social context in which they are not only accepted, but also accepted (Vitaro et al., 2007).

Each child has his or her own school-related experience; the differences of approach are the result of the individual approach or of the way parents' self-esteem was approached. A very important role is assigned to the emotional imprint the past has left on the child. Should there be positive emotional baggage, there are more chances that the child succeeds on a relational level and copes more easily with difficult events happening in their life.

Parents can strongly influence children's' self-esteem and the way they handle difficult situations. When children have difficulties in adapting themselves and relate to school-related situations, their parents' presence is important, so that they feel emotionally supported in learning how to overpass problems, manage things and find valuable life solutions. Furthermore, adults and teachers have a great impact on self-esteem; if they manage to help them, motivate them in difficult contexts, then self-esteem will win. "Teachers play a central role in a school's efforts to end bullying. According to trainers of the young minds, teachers are also the ones who shape the group ecologies, when teachers are warm and attentive to their students, they, in their turn, will no longer reject your colleagues as easily" (Whitson, 2017). This aspect also came from my observations and from the applied focus group.

In the preteen period, they are on the border between child and future adult. It can be the most delicate period with respect to emotions management, communication, especially due to the changes that preteens they undergo. All of these changes make preteens doubt themselves. Research has shown that, from a hormonal point of view, preteens experience an almost permanent troubled, sad and depressive mood. Many times, preteens lack the competence to live with this mood and that is why parents and teachers should be there to emotionally support them. 
A specific approach has to be taken according to different contexts of each child's age. Educational specialists and school counsellors carry out such specifically approached programs both for students, parents and teachers, but schools need more of them.

It is important to realize that the preteen stage implies a constant struggle on the intrapsychical level and this can lead to the occurrence of anxious, depressive, post-traumatic symptoms, as well as cognitive self, world and life distortions along with physical and physiological symptoms (more often conversions). The intensity of these symptoms can fluctuate, as preteens try to handle them and sometimes defence mechanisms succeed in holding them ground (and symptoms do not occur in the behavioural plan), but there are times when, due to the lack of self-control knowledge, their mechanisms fail in keeping the same efficiency (and symptoms occur on a behavioural level). Therefore, personal development programs brought in schools are imperative, since they have an impact on selfcontrol that preteens need so much.

Self-image represents ourselves in relation to the social environment. How do we see ourselves in society? How did we end up having these opinions about ourselves?

1.Social reflexion (it functions by mirror principle):

The social environment to which we belong proposes a definition and a value (reference point) for a particular feature. For example, you are cool if you experience undesirable behaviours...

the social group to which we belong "projects" on us (labels us), an image on what we are considered to be, an image that, once accepted and internalized, becomes a component element of the self-image.

2.Social comparison:

Even if a certain image is projected on them, people often feel the need for confirmation and reinforcement, social comparison providing the objective benchmarks needed to strengthen the self-image.

It is important to question ourselves: Who compares us and with whom? Who throws us all sorts of characters to whom we relate?

3.The role-play:

We are all social actors who play different roles on the stage of life. We do this on a daily basis and too many roles in a certain way can change us deeply.

Manipulate or not, we keep agitating on certain topics and we no longer recognize ourselves. Scientifically demonstrated, by accepting and interpreting a social role, there is a phenomenon of identification with the norms that regulate from a sociocultural point of view the specific behaviour of the respective role, while its characteristics are internalized and assumed.

So are the students with aggressive behaviour; even if they had not shown any aggressive behaviour in the past, they could change their behaviour through manipulation, playing different roles, which in fact do not represent them, but they believe that this is the only way that can survive through.

\section{THE INVESTIGATIVE APPROACH}

The initiation and development of the research approach of the relation between selfesteem and aggression took into account not only the objectives established for the research of this relationship, but also the specific characteristics of the group of people studied (preadolescent students) and the concrete conditions in which the research was carried out. 


\section{Research questions} esteem?

Could an intervention program reduce aggressive behaviours by developing self-

There is a correlation between aggressiveness and self-esteem?

\section{Purpose of the study}

Becoming aware of the increased level of manifestation of aggressive behaviours among children, as well as of the dramatic negative consequences that aggression carries over the children's health and balanced growth;

\section{Research objectives}

-Identifying the causes that lead to aggression;

-Identifying the factors that determine the increase of self-esteem;

-Becoming aware that an integrated school-family intervention is necessary for the correct and healthy management of aggressive behaviours;

\section{Research methods}

During the research, two tools have been applied in order to achieve the profile I had been aiming at, namely students with low/medium self-esteem and aggressive behaviour.

These tools are the Rosenberg Self-Esteem Questionnaire and the Buss-Perri Aggressive Behavior Questionnaire. These tools have been applied to 100 preteen students. Out of the 100 students, we identified 30 students that matched the search profile, whereof 15 students were involved in the study by applying the focus-group method.

In order to get information with respect to these questions, I have approached group focus method on preteen students from the Gymnasium School No.150, students who had the same problems, namely students with low or medium self-esteem, but with aggressive behaviours, as well. It is very important for the chosen group to be homogeneous, to have common characteristics so that discussions can take place more easily and be constructive. I chose this method because it is effective qualitative research as far as the relationship between results and duration is concerned, it is flexible in terms of the organization modalities and necessary resources, and the information received has a high degree of accuracy.

Within this focus group, the following significant aspects emerged for the research objectives. A low level of self-esteem relates to increased aggressiveness, especially through the forms of verbal aggression, anger, hostility.

By means of intervention activities, we tried to discuss with them what self-esteem means, how it is constructed, how important it is to have a positive perception about us. All respondents said that if they were heard, encouraged, appreciated or valued, they would be more balanced.

Students do not feel encouraged, they are punished by the family and teachers for any mistake and this leads to hostility from their side. Students with low self-esteem lack in trusting their own abilities, they cannot build proper self-esteem unless their parents and teachers trust them.

Students fail in trusting themselves because for the most part they are not accepted by the group of friends or colleagues and this aspect leads to undesirable behaviours, most of the time in order to get their existence noticed. This results further in joining a group that respects and accepts them but which also has a negative influence on them. 
Students feel neglected by their family members since they spend a lot of time at work. A consequence of neglecting students is frustration, the feeling that they lack their parents' love and frustration, in its turn, leads to aggressiveness.

Students made a top list of the causes of aggressive behaviour, namely: children are not listened to, they are not observed and encouraged by others, they are not valued, adults punish and discourage their initiatives. Students feel that counselling programs can often do wonders. Many of them have benefited by school counselling in the past and felt that these school counselling sessions lent wings to them.

When students were asked what they had felt when aggressiveness had come over them, a long list of other feelings came up, such as pain, disrespect, disappointment, worry, offence, rejection, frustration, disgust, shame, trauma.

By means of participative observation, I have carried out an activity of follow-up and systematic description of behaviours and events within the educational environment. As a school counselling teacher, I have aimed at an active engagement within the educational environment of students involved in this research and I have achieved a participative examination through in-class counselling activities deployed with the observed students.

Manipulative behaviours were observed. Within the counselling office, students were throwing out the mask they were wearing in class. We discussed this aspect within the focus group and it turned out that students needed this mask in the classroom, since they would be otherwise marginalized or even aggressed by the popular students in the class.

Anger, aggressive behaviour is actually a secondary emotion, being that often what we see on the surface can be misleading. We often tend to say that, practically, an aggressive student has high self-esteem, since he has the courage to be and act that way. As a matter of fact, that aggressive student comes with unstable emotional baggage.

There is a great need to sound a big alarm on this aspect and to try to discern the causes that led to that aggressive behaviour, actually intervening on the cause and not on the effect. Students have many different fears; most of them said they were afraid of failure, fear of rejection, fear of disappointment, fear of not being strong or good enough. Among this multitude of insights, we notice that their core is the "I-others" relationship because preteens try to discover while they discover themselves and it is normal for natural fears to arise in this context.

I also tried to experience intervention and I thought of intervention from children to children, considering that they relate to the core "I-others". In the counselling office, I invited students who talked about the problems they faced and the solutions they found and what were the solutions they found in relation to these problems. Several case studies were analyzed. The students were receptive and they said they did not feel alone anymore; they felt a sense of belonging and more than that they felt a strong motivation and the hope that they can overcome problems having more solutions at hand.

Significant importance must be conferred to prevention policies, the approach of each child according to his/her needs and especially the focus on group solutions, wherein everyone feels integrated, accepted and valued. The interventions are balanced, without judging, centred on everyone's comfort.

Thus, the acquired complex information provided clarifications on the studied issues and facilitated the identification of good practice examples for preteens' stimulation and personal, this aspect leading to a decrease in aggressive behaviours. We can mostly tell a person has high self-esteem proven by his/her behaviour but most of the times these persons 
show self-esteem in safety by his/her self-image insecurity or his heroics, narcissistic and defensive particularities. Following-up the relationship between self-esteem and aggression, it is important to observe, to show the significative differences for modern research on aggression, namely those between proactive and reactive aggression, direct and indirect aggression. This is why we aim ar analyzing the problem of self-esteem, its definition, its conditional factors and why it affects our lives so deeply.

During the difficult preteen period, the child needs emotional security, but, most of the time, the preteen does not show this. The way of expressing, affirming, imposing the personality does not always show us the true experiences since it often has a mask disguising many of their anxieties and fears. Often preteen oscillates between the feeling of power and the feeling of weakness and decreasing self-esteem. To protect themselves from these emotions, preteens develop reactions of provocation, aggression, opposition. Violence in school starts, first of all, from a communication deficiency; vulnerabilities and a whole lot of emotions are hidden because they think that, by revealing them, they will make a display of their vulnerabilities, troubles and the fear of being injured or marginalized. Fighting against school violence means improving the quality of relationships and communication between all persons involved in the educational area.

\section{CONCLUSION}

This research work has shown the need to investigate the beneficial effects of the counselling program on the overall self-esteem of low and medium self-esteem preteens and to track changes in aggressive behaviours. Also, this research will be expanded and it will be the basis of the experimental research I envisage within the doctoral school, the general objective being to highlight the need to develop and implement self-esteem training methods through the implementation of improvement intervention programs. See on the development of good practices, counselling programs, an option to prevent aggressive behaviours by increasing self-esteem, absolutely necessary for the good development of students.

As education specialists, we want to see that what we achieve has a real impact at the educational level. Student's emotional development is very important, hence we must intervene and draw attention to the aspects observed in the research. The attention should be both at the level of the family, teachers and the Ministry of Education in order to promote clear procedures and policies for specific intervention.

The research work has tried to draw the attention that focusing schools on cognitive development and neglecting other dimensions such as emotional or social is not an effective approach. Children's well-being means a balance between their needs for emotional stability and security, the needs to build quality social relationships and balanced self-esteem.

Emotional wounds hurt under different forms:

rejection, abandonment, anger, hatred, revenge, aggression, but human beings are so complex that no one has so far established what their limits really are. We are the limit, we strongly believe in this, we believe that when it hurts we can survive; it is not easy, it does not last long ... but it can help us to gro and discover another level yet not known to us. We see aggressive, uneducated, manipulative people ... the so-called unexplored people neither by others nor by themselves. One needs to travel to oneself; it is fascinating and painful at the same time, but we can only this way to maintain our mental health. We think it's important not to be afraid of relationships anymore; I think it is important to communicate, so that we could grow together, to help ourselves so that we should not have the feeling that we are 
alone and that, when finding ourselves at hard times, we have no one to call to. It is important to have the courage to look at those around us, appealing to compassion and curiosity ... so we should proceed with ourselves ... By means of socio-emotional development programs, we can start the journey to our inner self.

A child is not born violent, he learns violence. Some of the causes could be the exclusion of their group of friends, humiliation within their group of friends or in school. The attack actually means defence. Children defend themselves so as not to be victims, not to become victims and thus they become aggressors. Through aggressive behaviours, they validate their position as aggressors in the group.

What is to be done?

-The socio-emotional development programs in the school must be supported by parents;

-Legislative initiative on reducing aggression in schools should be implemented;

-A systematic approach should be implemented in order to decrease aggressive behaviours among children.

Children imitate everything they see; if they see aggression in their parents' behaviour or in the media, why are we surprised that they have become aggressive?

Considering that so many bullying cases are publicized, some of them with dramatic consequences such as deaths among children, public policy should be initiated urgently to prohibit bullying and take immediate measures against the aggressive ones and even against their parents if it is proven they have become aggressive due to their family environment.

When we refer to immediate measures, we do not refer to their punishment, as it is currently pursued, but we refer to the compulsory involvement of both aggressive students and their parents in socio-emotional development programs.

Researches lead to the conclusion that parents' aggressivity within the family leads to children's familiarization with aggression, consequently, its application. There are children who have only seen violence, disrespect and lack of tolerance in their family. They have not been taught how to connect to others.

Therefore, emotional and social education grant significance to what connection means, to their self-esteem development and connection to others and to the socio-emotional development. Why should we invest in children's socio-emotional development? Because these students will be the future adults with serious behaviour issues, those adults people fear, those adults who could even kill others. These programs no longer have to be options but urgencies where through procedures must be carried out so that to oblige the introduction of aggressive students in such programs. It is worth noting that among the criteria for establishing adult personality disorders, one of the criteria refers to the fact that the individual was involved in several acts of violence or had behavioural disorders during schooling. This aspect must be pursued.

Thus, socio-emotional development programs for students and parents is compulsory. The detection of these students prematurely, through collaboration with the family in granting the consent to enter such programs and through the collaboration with specialists can prevent many serious acts.

In this manner, the behaviours of future adults with personality disorders can be prevented. While interacting with aggressive behavioural students in the school counselling office, I noticed that all these students had problems in the family, as well, so one of the causes of their aggressive behaviour was related to family and family relationships. 
The family approach is important because it is relevant to take into account the significant relationships of the aggressive student. Therefore, we need to pay attention to the way family functions as an interconnected system, because it is known that when a wheel is affected, it is normal for this aspect to have repercussions on the whole. Often, what can greatly help the aggressive student to overcome his or her difficulties is related to the changes that occur within the family, the affective relationships between its members, the emotional availability that the family offers, the structure and the warmth that the child receives from the significant adults in his life. But when it comes to preteens, importance must be given both to the family, but especially to them. The focus is on the changes and transformations they can make in their life so that they can improve their aggression and their relationships.

If the greater investment is granted to socio-emotional education by creating more school counsellor positions that implement personal development programs, students' aggressive behaviours can be significantly reduced and prevented.

When education specialists decide that parents should help aggressive students, the goal is not to pity these students, but rather to understand why they have this aggressive behaviour and to use this information as a significant help in changing their behaviour. From my point of view, there is a paradox: aggressive students who try to intimidate others are actually those who most need others.

Aggression, just like bullying, is an acquired behaviour which was learnt at home, within the family, but the positive part is that it can be unlearnt. Children living in aggressive families need someone who can see beyond the noise of their violent behaviour and actually hear their cry for help. These children's deficit of emotional control drives them towards aggressive behaviours.

Scientific research does not always imply finding answers, but asking a great question. By analyzing and interpreting the collected data, they can be the basis for developing good practice examples in socio-emotional development. This product could be very valuable and useful for all teachers and education specialists and could be a landmark in understanding the relationship between self-esteem and aggressive behaviours. .

\section{BIBLIOGRAPHY:}

[1] Byrnes, J. (2008). Before conflict: Preventing aggressive behavior. Maryland: Rowman and Littlefield Education;

[2] Estevez, E., Jimenez, T., Moreno, D. (2018). „Aggressive behaviour in adolescence as a predictor of personal, family and school adjustment problems", Psichothema. 30:1, 66-73;

[3] Mennuti, R.B., Christner, W. , Freeman, A. (2016). Intervenții cogniniv-comportamentale în educatie, Ghid practic: ASCR;

[4] Vitaro, F., Boivin, M., Tremblay, R. (2007). „Peers and Violence: a Two-Sided Developmental Perspective, în Daniel Flannery, Alexander Vazsony, Irwin Waldman (coord.), The Cambridge Handbook of Violent Behaviour and Aggression. Cambridge: Cambridge University Press;

[5] Whitson, S. (2017). Fenomenul Bullying: 8 Strategii pentru a-i pune capăt, București: Editura Herald. 